\title{
Three active M dwarfs within 8 pc: L 449-1, L 43-72, and LP 949-15^
}

\author{
R.-D. Scholz ${ }^{1}$, G. Lo Curto ${ }^{2}$, R. A. Méndez ${ }^{3}$, V. Hambaryan ${ }^{1}$, E. Costa $^{3}$, T. J. Henry ${ }^{4}$, and A. D. Schwope ${ }^{1}$ \\ 1 Astrophysikalisches Institut Potsdam, An der Sternwarte 16, 14482 Potsdam, Germany \\ e-mail: [rdscholz; vhambaryan; aschwope]@aip.de \\ 2 European Southern Observatory, Alonso de Cordova 3107, Casilla 19001, Santiago 19, Chile \\ e-mail: glocurto@eso.org \\ 3 Departamento de Astronomía, Universidad de Chile, Casilla 36-D, Santiago, Chile \\ e-mail: [rmendez; costa]@das.uchile.cl \\ ${ }^{4}$ Department of Physics and Astronomy, Georgia State University, Atlanta, GA 30302-4106, USA \\ e-mail: thenry@chara.gsu.edu
}

Received 29 March 2005 / Accepted 11 May 2005

\begin{abstract}
Three nearby star candidates were selected as bright 2MASS point sources without associated optical sources, i.e. with potentially large proper motions, subsequently confirmed by multi-epoch optical data from the SuperCOSMOS Sky Surveys. All three objects are listed in the NLTT catalogue of high proper motion stars. Follow-up spectroscopic observations allowed us to classify L 449-1 as M 4.0e dwarf at 5.7 pc, L 43-72 as M 4.5e dwarf at 7.2 pc, and LP 949-15 as M 5.0e dwarf at $6.1 \mathrm{pc}$, using known absolute $J H K_{\mathrm{s}}$ magnitudes of dwarfs with corresponding spectral types, respectively. All three stars exhibit $\mathrm{H} \alpha$ emission lines, and all three can be identified with bright X-ray sources. The available ROSAT light curves of two of the objects show typical flare-like variability. Thus, all three stars are active, very close potential neighbours of the Sun, previously not mentioned as such, certainly deserving further attention. In particular, these are very promising candidates for trigonometric parallax programs.
\end{abstract}

Key words. surveys - astrometry - stars: kinematics - stars: low-mass, brown dwarfs - Galaxy: solar neighbourhood X-rays: stars

\section{Introduction}

The recently growing interest in getting the nearby stellar sample completed arises mainly from the fact that the nearest stars are excellent targets in search programs for extra-solar planets. In addition, the nearest stars of a given mass, spectral type, metallicity, age, etc. can be studied in most details and serve as benchmark sources in stellar physics. Finally, the solar neighbourhood offers the chance to study the stellar content and Galactic star formation history in a complete volume-limited sample.

Surprisingly, our knowledge of the solar neighbourhood is still lacking many stellar systems. Even in the immediate neighbourhood, within a $10 \mathrm{pc}$ horizon, where one may think that all stars have been registered, the number of missing systems is estimated between 25\% (Reid et al. 2003a) and more than $30 \%$ (Henry et al. 1997).

Many efforts have been started in recent years to fill the gaps. New sky surveys in the near-infrared, like the DEep Near-Infrared Survey (DENIS; Epchtein et al. 1997) and the

* Based on our systematic search in archival data from 2MASS and SuperCOSMOS Sky Surveys, and spectroscopic observations with the ESO NTT (ESO 072.D-0295).
Two-Micron All Sky Survey (2MASS; Cutri et al. 2003) and the completion of optical multi-colour and multi-epoch data bases like the SuperCOSMOS Sky Surveys (SSS; Hambly et al. 2001), and other archives provide powerful tools in the search for our unknown neighbours. The most spectacular discoveries, at approximate distances of only $4 \mathrm{pc}$, include some late-M dwarfs (Delfosse et al. 2001; Teegarden et al. 2003; Hambly et al. 2004; Henry et al. 2004) as well as the brown dwarf binary $\varepsilon$ Indi Ba,Bb (Scholz et al. 2003; McCaughrean et al. 2004).

High proper motion catalogues, such as the New Luyten Two Tenths (NLTT) catalogue (Luyten 1979-80) do also still play an important role in the finding of new nearby stars, especially if combined with new accurate photometry, e.g. from 2MASS and spectroscopy (Scholz et al. 2001; Reid \& Cruz 2002; Cruz \& Reid 2002; Reid et al. 2002; McCaughrean et al. 2002; Reid et al. 2003b; Scholz et al. 2005).

In this short paper we present further three NLTT stars as newly recognised close neighbours. Section 2 describes the selection of the candidates. Section 3 presents optical classification spectroscopy and corresponding distance determination. Section 4 summarises the available X-ray data; and in Sect. 5 we briefly discuss the results. 


\section{Selection of nearby candidates}

Nearby candidates in the southern sky were first detected as proper motion objects from comparing four different epoch observations in the SSS at the positions of bright $(J<14) 2 \mathrm{MASS}$ point sources without optical identification. The initial sample of candidates was the same as described in Scholz et al. (2004), i.e. 11000 objects outside the Galactic plane. There is a high probability that an object without optical counterpart (within 5 arcsec) in the 2MASS data base has a large proper motion ( $>0.3 \mathrm{arcsec} / \mathrm{yr}$ ), since the epoch difference between 2MASS and the USNO A2.0 catalogue (Monet et al. 1998) used for the cross-identification is typically about 15 years at southern declinations. If a candidate was confirmed as moving object during the visual inspection, then its accurate proper motion was determined using all available epochs from the SSS, 2MASS and DENIS. The three objects listed in Table 1 were by far the brightest 2MASS sources identified in this way as proper motion objects, which had no previous distance estimates. Some SSS and DENIS magnitudes given in Table 1 represent mean values from different epochs. More details can be found in the notes below the table.

A query in VizieR $^{1}$ allowed us to identify the three stars with entries in the NLTT and with bright X-ray sources: L 449-1 = 1RXS J051723.3-352152, LP 949-15 = 1RXS J060452.1-343331, and L 43-72 = 1RXS J181116.4-785919 (Luyten 1979-80; Voges et al. $1999)$, although we note a large positional error ( 90 arcsec) in the NLTT for L 449-1.

\section{Spectroscopy, distances, and kinematics}

Spectroscopic observations of L 449-1, L 43-72, and LP 949-15, and of two comparison stars, Gl 65B (M 6.0) and G1 213 (M 4.0), were carried out with the EMMI instrument at the $3.5 \mathrm{~m}$ New Technology Telescope (NTT), ESO La Silla, on October 4th, 2003. We used grism\#4 and a 1 arcsec slit yielding a spectral dispersion of $1.76 \AA /$ pix. With the $2 \times 2$ binning used, this corresponds to a resolution of about 600 . The total exposure times for these very bright stars were less than $1 \mathrm{~s}$.

The reduction of the raw spectra was carried out using standard ESO MIDAS routines, including an optimum extraction, cosmics removal, and extinction correction. Fluxes were calibrated using standard stars (LTT 7379 and LTT 2415) measured close in time to the objects. The fluxes of L 449-1 and LP 949-15 are better determined than the one for L 43-72 since the former were observed at the end of the night, when weather conditions were much more stable. L 43-72 was observed at relatively high air mass (1.65) due to its position on the sky. The fully calibrated spectra normalised at $7500 \AA$ are shown in Fig. 1, with the two well known comparison stars, Gl 65B and G1 213 on top and at the bottom of the plot, respectively.

We measured the spectral indices TiO5 and PC3 and obtained spectral types using the formulae given in Reid et al. (1995) and Martín et al. (1999), respectively (Table 2). The additional classification from the visual comparison with the spectra of Gl 65B and Gl 213 is also given in the table.

\footnotetext{
${ }^{1}$ http://vizier.u-strasbg.fr/.
}

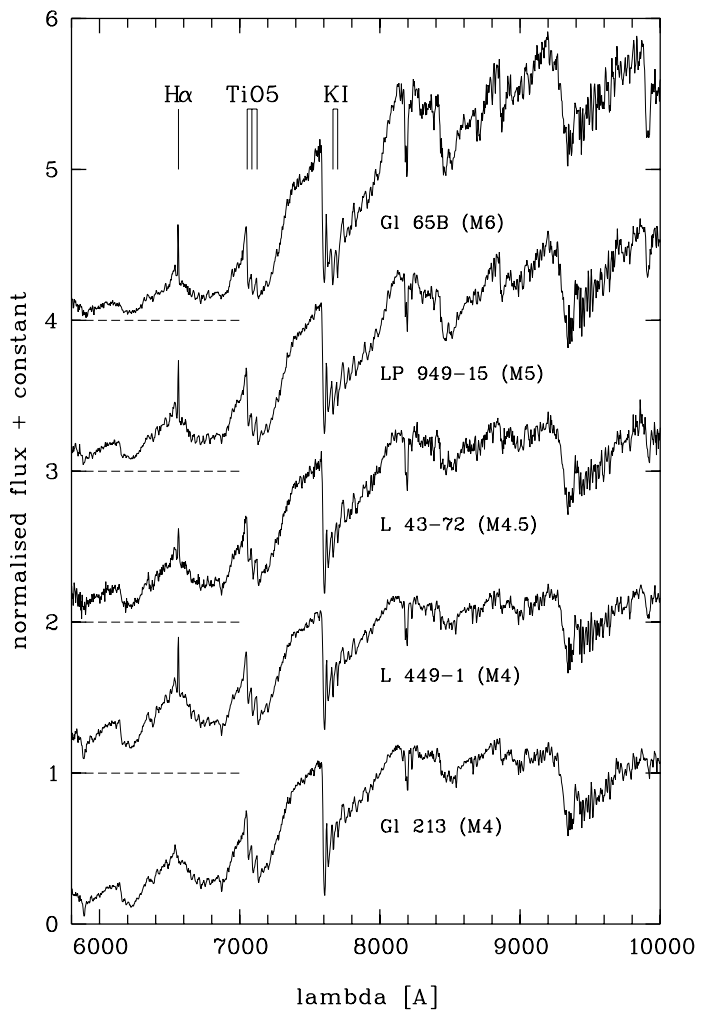

Fig. 1. Optical spectra taken with EMMI on the ESO NTT. The two comparison star spectra, obtained in the same run, are shown on top and at the bottom. Features used for a rough measurement of the radial velocities are labeled.

The finally adopted spectral type corresponds to the mean of the latter three estimates, rounded to half a spectral sub-type. The visual comparison clearly indicates a later spectral type for LP 949-15 than for L 43-72, as can also be concluded from the TiO5 measurements.

Distances were estimated using the 2MASS $J H K_{\mathrm{s}}$ magnitudes from Table 1 and mean absolute magnitudes of M 4.0 to M 5.0 dwarfs from Kirkpatrick \& McCarthy (1994), conservatively assuming uncertainties of 0.6 mag in the absolute magnitudes. This roughly corresponds to the maximum $M_{J}$ dispersion at spectral type $\mathrm{M} 4.0$, which is even larger than the $\sim 0.5$ mag difference in $M_{J}$ between stars deviating by half a spectral sub-class in the range of M 3.5 to M 5.5 (Scholz et al. 2005). All three stars fall in the 8 pc limit (Table 2). The results of alternative distance estimates, given in the Notes to Table 2, do not deviate significantly from those listed in the table.

Rough estimates of the radial velocities were obtained by measuring the shift of the $\mathrm{H} \alpha$ emission line, the KI doublet, and sharp TiO5 features (marked in Fig. 1) with respect to the two comparison spectra taken during the same night. The radial velocities of the comparison stars G1 213 and Gl65B are known as $+104.3 \mathrm{~km} \mathrm{~s}^{-1}$ and $+29.0 \mathrm{~km} \mathrm{~s}^{-1}$, respectively (Barbier-Brossat $\&$ Figon 2000). The relative measurements were corrected for the Earth's motion. The resulting mean radial velocities with an accuracy of about $30 \mathrm{~km} \mathrm{~s}^{-1}$ are listed in Table 2 .

Combining all the available information we computed heliocentric space motions following Johnson \& Soderblom (1987). The $U V W$ velocity components of all three stars are 
Table 1. Astrometry and photometry from the 2MASS, SSS, and DENIS.

\begin{tabular}{|c|c|c|c|c|c|c|c|c|c|c|}
\hline Name & $\begin{array}{c}\alpha, \delta \\
(\mathrm{J} 2000)\end{array}$ & Epoch & \multicolumn{2}{|c|}{$\mathrm{mas} / \mathrm{yr}$} & (SSS) & S) $R$ & $\begin{array}{c}I \\
(\mathrm{DENIS})^{d}\end{array}$ & $\bar{J}$ & $\begin{array}{c}H \\
\text { 2MASS) }\end{array}$ & $\overline{K_{\mathrm{s}}}$ \\
\hline L 449 & $51722.92-352154.5$ & 199 & $-214 \pm 4$ & -16 & 13.023 & 10.743 & 8.897 & 7.400 & 6.854 & 6.558 \\
\hline LP 94 & $060452.15-34$ & & & & 13.784 & & 9.027 & 7.742 & 7.183 & 6.866 \\
\hline $\mathrm{L} 43-72^{c}$ & $181115.28-785922.7$ & 2000.378 & $+83 \pm 7$ & $+308 \pm 9$ & 13.736 & 11.504 & 9.490 & 7.840 & 7.327 & 6.964 \\
\hline
\end{tabular}

Notes: positions are from 2MASS, which provided accurate astrometry at the most recent epochs. Further details are given below.

${ }^{a} \mu$ from 1 2MASS, 1 DENIS, and 4 SSS positions; two SSS $R$ magnitudes, OR $=10.697$ and $\mathrm{RE}=10.789$, were averaged; DENIS $J=$ $7.262, K=6.484$.

${ }^{b} \mu$ from $12 \mathrm{MASS}$, and 4 SSS positions; two SSS $R$ magnitudes, $\mathrm{OR}=11.702$ and $\mathrm{RE}=11.455$, were averaged.

${ }^{c} \mu$ from 1 2MASS, 3 DENIS, and 5 SSS positions; mean DENIS $J=7.811, K=7.023 ; R$ magnitude $=$ RE, two other strongly deviating SSS $R$ magnitudes, $\mathrm{OR}=15.348(?)$ and $\mathrm{OR}=16.470($ ?), were not used.

${ }^{d}$ For L 43-72, the mean of three independent, very similar measurements is given. In case of LP 949-15 DENIS data were not available so that the SSS I magnitude is listed instead. The SSS I magnitudes of L 449-1 and L 43-72 are 8.643 and 9.635, respectively.

Table 2. Spectral indices and types, radial velocities, distances, and heliocentric space motions. Radial velocity errors are about $30 \mathrm{~km} \mathrm{~s}^{-1}$.

\begin{tabular}{lcccccccccccc}
\hline \hline Name & TiO5 & PC3 & $\mathrm{SpT}_{\mathrm{TiO} 5}$ & $\mathrm{SpT}_{\mathrm{PC} 3}$ & $\mathrm{SpT}_{\text {comp }}$ & $\mathrm{SpT}_{\text {final }}$ & $\begin{array}{c}d_{\text {spec }+J H K_{\mathrm{S}}} \\
{[\mathrm{pc}]}\end{array}$ & $\begin{array}{c}v_{\text {tan }} \\
{\left[\mathrm{km} \mathrm{s}^{-1}\right]}\end{array}$ & $\begin{array}{c}v_{\text {rad }} \\
{\left[\mathrm{km} \mathrm{s}^{-1}\right]}\end{array}$ & $\begin{array}{c}U \\
{\left[\mathrm{~km} \mathrm{~s}^{-1}\right]}\end{array}$ & $\begin{array}{c}V \\
{\left[\mathrm{~km} \mathrm{~s}^{-1}\right]}\end{array}$ & $\begin{array}{c}W \\
{\left[\mathrm{~km} \mathrm{~s}^{-1}\right]}\end{array}$ \\
\hline L 449-1 & 0.422 & 1.081 & M 3.7 & M 3.6 & M 4.0 & M 4.0 & $5.7 \pm 1.2$ & 7 & +13 & $-1 \pm 13$ & $-8 \pm 22$ & $-13 \pm 17$ \\
LP 949-15 & 0.301 & 1.171 & M 5.0 & M 4.3 & M 5.0 & M 5.0 & $6.1 \pm 1.3$ & 10 & +67 & $-38 \pm 13$ & $-51 \pm 24$ & $-24 \pm 12$ \\
L 43-72 & 0.393 & 1.204 & M 4.0 & M 4.5 & M 4.5 & M 4.5 & $7.2 \pm 1.5$ & 11 & +66 & $+51 \pm 19$ & $-37 \pm 19$ & $-23 \pm 13$ \\
\hline
\end{tabular}

Notes: for L 449-1 and L 43-72 we can use the DENIS IJK photometry (Table 1) for an alternative distance determination using the absolute magnitudes of M 4.0 and M 4.5 dwarfs given in Kirkpatrick \& McCarthy (1994). The results of 5.5 pc (L 449-1) and 7.2 pc (L 43-72) are almost in exact agreement with those obtained from the 2MASS photometry. Using the absolute magnitudes $M_{J}=8.37,8.85,9.30$, derived by Scholz et al. (2005) for M 4.0, M 4.5, and M 5.0 dwarfs, respectively, we obtain distances of 6.4 pc, 6.3 pc, and 4.9 pc, for L 449-1, L 43-72, and LP 949-15, respectively. The combination of the photographic SSS and 2MASS magnitudes (Hambly et al. 2004) yields 6.5 pc, $6.1 \mathrm{pc}$, and 6.7 pc for L 449-1, LP 949-15, and L 43-72, respectively.

not very significant due to the relatively large errors. However, such small motions are typical of the Galactic thin disk population.

\section{X-ray data}

All three objects were detected as bright X-ray sources in the ROSAT all-sky survey (Voges et al. 1999), with mean count rates between 0.4 and 0.6 counts per second. Two of the sources have long enough exposure times of approximately $600 \mathrm{~s}$ so that their light curves can be plotted (Figs. 2 and 3). These two objects were already mentioned as variable X-ray sources by Fuhrmeister \& Schmitt (2003). Their light curves show very significant variations, which can be interpreted as flares, perhaps with associated irregular X-ray brightness variations of comparatively lower amplitudes.

L 449-1 and LP 949-15 were already mentioned as dMe counterparts of ROSAT Wide Field Camera (WFC) sources by Pounds et al. (1993). X-ray variability was not detected in the ROSAT WFC data (McGale et al. 1995), most likely due to the much poorer count rate statistics. All three objects are also listed as EUVE sources (e.g. Malina et al. 1994; Bowyer et al. 1996). LP 949-15 appears in the QORG catalogue of radio/X-ray sources by Flesch \& Hardcastle (2004).

Our estimates of X-ray and bolometric luminosities yielded $\log \left(L_{\mathrm{X}} / L_{\mathrm{bol}}\right) \approx-4.0 \ldots-3.0$ for the three stars, where the lower and higher values correspond to the mean and maximum level of the light curves, respectively. These results are in good

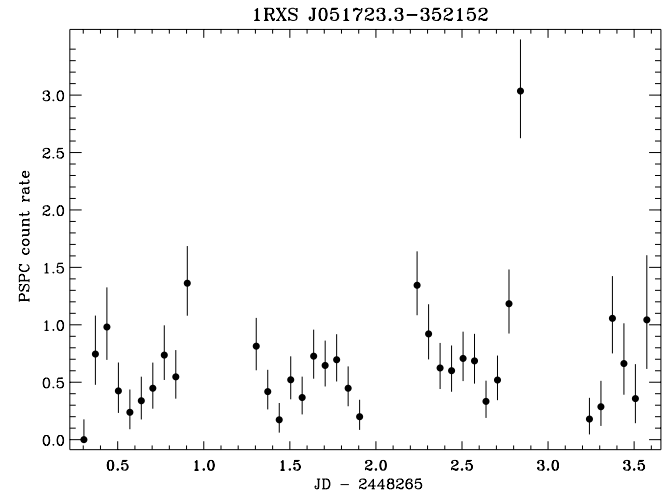

Fig. 2. Light curve from ROSAT All Sky Survey observations of 1RXS J051723.3-352152, which we identified with L 449-1. Shown are the background subtracted and vignetting corrected count rates as a function of time.

agreement with those for other M 4.0-M 5.0 dwarfs (see, for example, Fig. 5 in Hambaryan et al. 2004).

\section{Discussion}

LP 949-15 is listed as AP Col, a variable star of UV Cet type, in the General Catalog of Variable Stars (Samus et al. 2004). The amplitude of its variability in the $V$ band is given as 2.5 mag. The available SSS photometry (two independent $R$ magnitudes of 11.70 and 11.45, respectively), the SSS colours (Table 1, and the additional photometry $(V=13.17)$ from the Carlsberg 


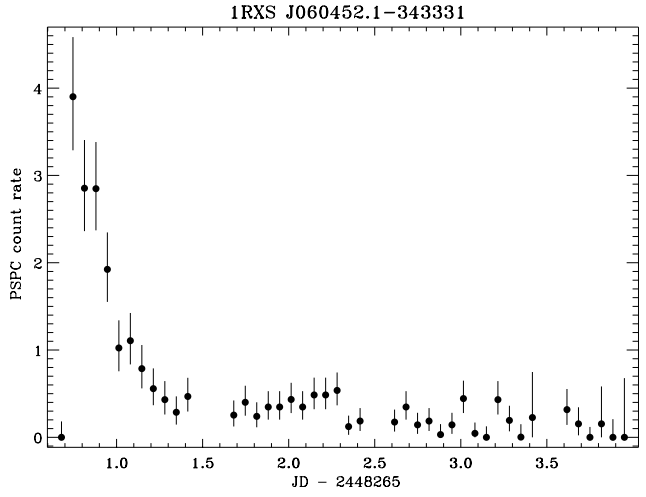

Fig. 3. Same as in Fig. 2, but for 1RXS J060452.1-343331 identified with LP 949-15.

Meridian Catalogs (CMC 1999) do not hint at strong variability. In addition, our distance estimate based on the near-infrared photometry $(6.1 \mathrm{pc})$, is in reasonable good agreement with alternative estimates based on the CMC $V$ magnitude $(7.0 \mathrm{pc}$ ) and SSS $I$ magnitude $(4.8 \mathrm{pc})$, if we use again absolute magnitudes of M 5.0 dwarfs from Kirkpatrick \& McCarthy (1994). Therefore, we think that our distance estimates for LP 949-15 are not strongly biased by its flare activity. Even taking into account the relatively large uncertainties in our distance estimates, all three stars are very likely to be within $10 \mathrm{pc}$.

L 449-1 was previously thought to be a carbon star (Alksnis et al. 2001), which is ruled out by our findings. Other interesting cross-identifications include those of L 449-1 and L 43-72 with the IRAS faint source catalogue (Moshir et al. 1989).

Although all three stars are known X-ray sources and were earlier classified as proper motion stars, we are not aware of any publication treating them as nearby stars. Our crossidentification with the 2MASS providing accurate photometry and the follow-up low-resolution spectroscopy allowed us to uncover them as close neighbours to the Sun. As such, they are certainly worth investigating further, with more accurate distances to be obtained in a trigonometric parallax program.

Note added in proof: Thierry Forveille (private communication) draw our attention to the fact that L 43-72 was previously mentioned as a nearby $(d \sim 9 \mathrm{pc})$ star by Polomski et al. (1997).

Acknowledgements. Our search for nearby star candidates was primarily based on data from the Two-Micron All Sky Survey, a joint project of the University of Massachusetts and the Infrared Processing and Analysis Center/California Institute of Technology, funded by the NASA and NSF, and from the SuperCOSMOS Sky Surveys at the Wide-Field Astronomy Unit of the Institute for Astronomy, University of Edinburgh. The spectroscopic observations were carried out with the ESO NTT. We acknowledge the use of the Simbad database and the VizieR Catalogue Service operated at the CDS. E.C. and R.A.M. acknowledge support by the Fondo Nacional de Investigación Científica y Tecnológica (proyecto No. 1010137, Fondecyt) and by the Chilean Centro de Astrofísica FONDAP (No. 15010003). We thank the referee, Neill Reid, for his prompt report and helpful comments.

\section{References}

Alksnis, A., Balklavs, A., Dzervitis, U., et al. 2001, Baltic Astron., 10,1
Barbier-Brossat, M., \& Figon, P. 2000, A\&AS, 142, 217

Bowyer, S., Lampton, M., Lewis, J., et al. 1996, ApJS, 102, 129

CMC 1999, Carlsberg Meridian Catalogs Number 1-11, Copenhagen University Obs., Royal Greenwich Obs., and Real Instituto y Observatorio de la Armada en San Fernando, http://vizier.u-strasbg.fr/viz-bin/Cat?I/256

Cruz, K. L., \& Reid, I. N. 2002, AJ, 123, 2828

Cutri, R. M., Skrutskie, M. F., Van Dyk, S., et al. 2003, The 2MASS All-Sky Catalog of Point Sources, University of Massachusetts and IPAC/California Institute of Technology

Delfosse, X., Forveille, T., Martín, E. L., et al. 2001, A\&A, 366, L13

Epchtein, N., de Batz, B., Capoani, L., et al. 1997, Msngr, 87, 27

Flesch, E., \& Hardcastle, M. J. 2004, A\&A, 427, 387

Fuhrmeister, B., \& Schmitt, J. H. M. M. 2003, A\&A, 403, 247

Hambaryan, V., Staude, A., Schwope, A. D., et al. 2004, A\&A, 415, 265

Hambly, N. C., MacGillivray, H. T., Read M. A., et al. 2001, MNRAS, 326,1279

Hambly, N. C., Henry, T. J., Subasavage, J. P., et al. 2004, AJ, 128, 437

Henry, T. J., Ianna, P. A., Kirkpatrick, J. D., \& Jahreiß, H. 1997, AJ, 114,388

Henry, T. J., Subasavage, J. P., Brown, M. A., et al. 2004, AJ, 128, 2460

Johnson, D. R. H., \& Soderblom, D. R. 1987, AJ, 93, 864

Kirkpatrick, J. D., \& McCarthy, D. W. 1994, AJ, 107, 333

Luyten W. J., 1979-1980, New Luyten Catalogue of Stars with Proper Motions Larger than Two Tenths of an Arcsecond (Minneapolis: Univ. Minnesota), ADC Selected Astronomical Catalogs, 1, CD-ROM

Malina, R. F., Marshall, H. L., Antia, B., et al. 1994, AJ, 107, 751

Martín, E. L., Delfosse, X., Basri, G., et al. 1999, AJ, 118, 2466

McCaughrean, M. J., Scholz, R.-D., \& Lodieu, N. 2002, A\&A, 390, L27

McCaughrean, M. J., Close, L. M., Scholz, R.-D., et al. 2004, A\&A, 413, 1029

McGale, P. A., Allan, D. J., Barber, C. R., et al. 1995, AdSpR, 16, 69

Monet, D., Bird, A., Canzian, B., et al. 1998, USNO-A V2.0, A Catalog of Astrometric Standards, US Naval Observatory Flagstaff Station (USNOFS) and Universities Space Research Association (USRA) stationed at USNOFS

Moshir, M., Copan, G., Conrow, T., et al. 1989, IRAS Faint Source Catalog, $|b|>10$ Degrees, Version 2.0, Infrared Processing and Analysis Center

Polomski, E., Vennes, S., Thorstensen, J. R., Mathioudakis, M., Falco, E. E. 1997, ApJ, 486, 179

Pounds, K. A., Allan, D. J., Barber, C. et al. 1993, MNRAS, 260, 77

Reid, I. N., \& Cruz, K. L. 2002, AJ, 123, 2806

Reid, I. N., Hawley, S. L., \& Gizis, J. E. 1995, AJ, 110, 1838

Reid, I. N., Kilkenny, D., \& Cruz, K. L. 2002, AJ, 123, 2822

Reid, I. N., Cruz, K. L., Laurie S. P., et al. 2003a, AJ, 125, 354

Reid, I. N., Cruz, K. L., Allen, P., et al. 2003b, AJ, 126, 3007

Samus, N. N., Durlevich, O. V., et al. 2004, Combined General Catalog of Variable Stars (GCVS4.2), Institute of Astronomy of Russian Academy of Sciences and Sternberg State Astronomical Institute of the Moscow State University, http://vizier.u-strasbg.fr/viz-bin/Cat?II/250

Scholz, R.-D., Meusinger, H., \& Jahreiß, H. 2001, A\&A, 374, L12

Scholz, R.-D., McCaughrean, M. J., Lodieu, N., \& Kuhlbrodt, B. 2003, A\&A, 398, L29

Scholz, R.-D., McCaughrean, M. J., \& Lodieu, N. 2004, A\&A, 428, L25

Scholz, R.-D., Meusinger, H., \& Jahreiß, H. 2005, A\&A, submitted Teegarden, B. J., Pravdo, S. H., Hicks, M., et al. 2003, ApJ, 589, L51 Voges, W., Aschenbach, B., Boller, T., et al. 1999, A\&A, 349, 389 